\title{
On the critical points of the energy functional on vector fields of a Riemannian manifold
}

\author{
Giovanni Nunes Jaime Ripoll
}

February 11, 2017

\begin{abstract}
Given a compact Lie subgroup $G$ of the isometry group of a compact Riemannian manifold $M$ with a Riemannian connection $\nabla$, it is introduced a $G$-symmetrization process of a vector field of $M$ and it is proved that the critical points of the energy functional

$$
F(X):=\frac{\int_{M}\|\nabla X\|^{2} d M}{\int_{M}\|X\|^{2} d M}
$$

on the space of $G$-invariant vector fields are critical points of $F$ on the space of all vector fields of $M$, and that this inclusion may be strict in general. One proves that the infimum of $F$ on $\mathbb{S}^{3}$ is not assumed by a $\mathbb{S}^{3}$-invariant vector field. It is proved that the infimum of $F$ on a sphere $\mathbb{S}^{n}, n \geq 2$, of radius $1 / k$, is $k^{2}$, and is assumed by a vector field invariant by the isotropy subgroup of the isometry group of $\mathbb{S}^{n}$ at any given point of $\mathbb{S}^{n}$. It is proved that if $G$ is a compact Lie subgroup of the isometry group of a compact rank 1 symmetric space $M$ which leaves pointwise fixed a totally geodesic submanifold of dimension bigger than or equal to 1 then all the critical points of $F$ are assumed by a $G$-invariant vector field.

Finally, it is obtained a characterization of the spheres by proving that on a certain class of Riemannian compact manifolds $M$ that contains rotationally symmetric manifolds and rank 1 symmetric spaces, with positive Ricci curvature $\operatorname{Ric}_{M}, F$ has the lower bound $\operatorname{Ric}_{M} /(n-1)$ among the $G$ - invariant vector fields, where $G$ is the isotropy subgroup of the isometry group of $M$ at a point of $M$, and that his lower bound is attained if and only if $M$ is a sphere of radius $1 / \sqrt{\mathrm{Ric}_{M}}$.
\end{abstract}

\section{Introduction}

Let $M$ be a compact, orientable, $n$-dimensional, $n \geq 2, C^{\infty}$ manifold with a Riemannian metric and let $\nabla$ be the Riemannian connection of $M$. 
In this paper we study the critical points of the energy of $\nabla$ acting on the space $C^{\infty}(T M)$ of $C^{\infty}$ vector fields of $M$ with unit $L^{2}$ norm. Precisely, we study the critical points of the functional

$$
F(X)=\int_{M}\|\nabla X\|^{2} d M
$$

on the space of vector fields $X \in C^{\infty}(T M)$ such that

$$
\int_{M}\|X\|^{2} d M=1
$$

It is well known that the critical values of $F$ are the eingenvalues of the so called rough Laplacian $-\operatorname{div} \nabla$ of $M$ [1] and it follows from the spectral theory for linear elliptic operators that they constitute an increasing sequence $0 \leq \delta_{1}<\delta_{2}<\cdots \rightarrow+\infty$ (counted with multiplicity) which are assumed by $C^{\infty}$ eigenvector fields. Moreover, if $M$ has no parallel vector fields then the infimum $\delta_{1}$ of $F$ is positive.

The search of geometric estimates of the spectrum of elliptic linear operators is an active topic of investigation in Geometric Analysis, the Laplacian being already a classical and well studied one [10]. As to the study of the rough Laplacian operator, it seems to the authors that no attention has been paid so far. We have not found in the literature a description of its eigenvalues even in the simplest Riemannian spaces as the spheres.

In this paper we study the critical points of $F$ on Riemannian compact manifolds admitting a nontrivial isometry group. A simple but fundamental idea here is to introduce a process of symmetrization of a vector field of $M$ by a compact Lie subgroup $G$ of the isometry group $\operatorname{Iso}(M)$ of $M$. We then use this symmetrization to prove that, apart some exceptional cases where the symmetrization process leads to zero vector fields, the critical points of $F$ on the space of $G$-invariant vector fields are in the spectrum of $F$ (Proposition 1). What is somewhat surprising is that this inclusion may be strict, even for the infimum: We prove that the infimum of $F$ in the unit sphere $\mathbb{S}^{3}$ is not realized by a $\mathbb{S}^{3}$ - invariant vector field, considering $\mathbb{S}^{3}$ as a Lie subgroup of $O(4)$ (Remark 3); in other words, left invariant vector fields of $\mathbb{S}^{3}$ (with a bi-invariant metric) are critical points of $F$ but are not ones with least energy. This raises the problem of whether the infimum or more generally the spectrum of $F$ is assumed by $G$-invariant vector fields for a given compact Lie subgroup $G$ of the isometry group of $M$.

We prove that the orthogonal projection $V$ of a nonzero vector $v$ of $\mathbb{R}^{n+1}$ on $T \mathbb{S}^{n}(1 / k)$, where $\mathbb{S}^{n}(1 / k)$ is a sphere of radius $1 / k$, realizes the 
infimum of $F$ on $C^{\infty}\left(T \mathbb{S}^{n}(1 / k)\right)$ and that this infimum is $k^{2}$ (Theorem 4) (we note that $V$ is invariant by the isotropy subgroup of $O(n+1)$ that leaves fixed the point $\left.v /(k|v|) \in \mathbb{S}^{n}(1 / k)\right)$. Moreover, we prove that if $X$ is a Killing field of $\mathbb{S}^{n}(1 / k)$ then $F(X)=(n-1) k^{2}$. It then follows that $X$ minimizes the energy if and only if $n=2$. As a consequence, if $\mathcal{S}_{n} \subset$ $C^{\infty}\left(T \mathbb{S}^{n}(1 / k)\right)$ denotes the space of eigenvector fields associated to $k^{2}$ then $\operatorname{dim} \mathcal{S}_{2} \geq \operatorname{dim} O(3)+\operatorname{dim} \mathbb{R}^{3}=6$. By using Fourier series it is proved in [2] that actually $\operatorname{dim} \mathcal{S}_{2}=6$.

We also prove that if $M$ is a compact rank 1 symmetric space and if $G$ is a Lie subgroup of Iso $(M)$ which leaves pointwise fixed a totally geodesic submanifold of dimension bigger than or equal to 1 then all the critical points of $F$ are realized by a $G$-invariant vector fields (Theorem 9). We note that there are many subgroups $G$ of $\operatorname{Iso}(M)$ satisfying this condition. For example, any totally geodesic $m$-dimensional submanifold of $\mathbb{S}^{n}(1 / k)$, $1 \leq m \leq n-2$, is the fixed point of a compact subgroup of $O(n+1)$ isomorphic to $O(n-m)$. More generally, any rank 1 symmetric space is plenty of totally geodesic submanifolds all of them being the fixed points of some compact Lie subgroup of the isometry group of the space (see [4]). We also mention the groups of reflections on totally geodesic submanifolds, which are finite groups isomorphic to $\mathbb{Z}_{2}$.

In the last part of the paper we obtain a characterization of the sphere as a space where $F$ attains the infimum of the energy on a certain class of Riemannian manifolds as explained next. We shall say that $M$ is two point symmetric with center $p \in M$ if the isotropy $\operatorname{subgroup} \operatorname{Iso}_{p}(M)$ of $\operatorname{Iso}(M)$ at $p$ is isomorphic to the isotropy subgroup of the isometry group of a rank 1 symmetric space, that is, $\operatorname{Iso}_{p}(M)$ is isomorphic to some of the following Lie groups: $O(n), U(1) \times U(n-1), S p(1) \times S p(n-1)$ or $\operatorname{Spin}(9)$ (see [4] and Definition 11 ahead). Note that when $\operatorname{Iso}_{p}(M)=O(n)$ then $M$ is a rotationally symmetric space (see [3]). Symmetric spaces of rank 1 satisfy the so called two point homogeneous property and hence are also known as two point homogeneous spaces.

We prove that if $M$ is a two point symmetric space with center $p$ and with Ricci curvature $\operatorname{Ric}_{M}$ satisfying $\operatorname{Ric}_{M} \geq(n-1) k^{2}$, then the infimum of $F$ on the space of $\operatorname{Iso}_{p}(M)$-invariant vector fields is bigger than or equal to $k^{2}$ and the equality holds if and only if $M$ is a sphere of radius $1 / k$ (Theorem 12). 


\section{A general result}

Let $M$ be a compact Riemannian manifold of dimension $n \geq 2$. We choose on the full isometry group Iso $(M)$ of $M$ a fixed left invariant Riemannian metric. We consider on any Lie subgroup of Iso $(M)$ the left invariant Riemannian metric induced by the one of Iso $(M)$.

Let $G$ be a compact Lie subgroup of Iso $(M)$. Given a vector field $V \in$ $C^{\infty}(T M)$, the $G$-symmetrization of $V$ is the vector field $V_{G}$ defined by setting, at a given $p \in M$,

$$
\left\langle V_{G}(p), u\right\rangle=\frac{1}{\operatorname{Vol}(G)} \int_{G}\left\langle\left(d g_{p}\right)^{-1} V(g(p)), u\right\rangle d g
$$

where $u \in T_{p} M$. We note that $G$ may be finite case in which $V_{G}$ is given by

$$
V_{G}(p)=\frac{1}{|G|} \sum_{g \in G} d g_{p}^{-1}(V(g(p)),
$$

where $|G|$ denotes the number of elements of $G$. Note that $V_{G} \in C^{\infty}(T M)$ is $G$-invariant, that is, $V_{G}(g(p))=d g_{p}\left(V_{G}(p)\right)$ for all $p \in M$ and $g \in G$. Moreover, by the linearity of $\operatorname{div} \nabla$ and of the integration process we have

$$
(\operatorname{div} \nabla V)_{G}=\operatorname{div} \nabla V_{G}
$$

In particular, if $V$ satisfies $\operatorname{div} \nabla V=-\lambda V$ then $\operatorname{div} \nabla V_{G}=-\lambda V_{G}$. We call $V_{G}$ the $G$-mean of $V$.

We observe that depending on $M$ and $G$ it may happen that $V_{G} \equiv 0$. This happens with any vector field $V$ on a rank 1 compact symmetric space $M$ if $G$ is the full isometry group of isometries of $M$ (this is consequence of Lemma 7). We prove:

Proposition 1 Let $M$ be a compact $n$-dimensional Riemannian manifold and $G$ a compact Lie subgroup of Iso $(M)$. Then the eigenvalues and eigenvectors of $F$ restrict to the subspace of $G$ - invariant vector fields of $C^{\infty}(T M)$ are also eigenvalues and eigenvectors of $F$ on $C^{\infty}(T M)$.

We need the following result:

Lemma 2 On the hypothesis of the Proposition 1 assume that $W \in C^{\infty}(T M)$ satisfies $W_{G} \equiv 0$. Let $V$ be a $G$-invariant vector field. Then

$$
\int_{M}\langle W(x), V(x)\rangle d x=0 .
$$


Proof. Since the elements of $G$ are isometries and $V$ is $G$-invariant we have, for all $g \in G$

$$
\begin{aligned}
\int_{M}\langle W(x), V(x)\rangle d x & =\int_{M}\langle W(g(x)), V(g(x))\rangle d x \\
& =\int_{M}\left\langle d g_{x}^{-1}(W(g(x))), d g_{x}^{-1}(V(g(x)))\right\rangle d x \\
& =\int_{M}\left\langle d g_{x}^{-1}(W(g(x))), V(x)\right\rangle d x .
\end{aligned}
$$

It follows from Fubini's theorem that

$$
\begin{aligned}
\int_{M}\langle W(x), V(x)\rangle d x & =\frac{1}{\operatorname{Vol}(G)} \int_{G} \int_{M}\left\langle d g_{x}^{-1}(W(g(x))), V(x)\right\rangle d x d g \\
& =\int_{M}\left\langle\frac{1}{\operatorname{Vol}(G)} \int_{G} d g_{x}^{-1}(W(g(x))) d g, V(x)\right\rangle d x \\
& =\int_{M}\left\langle W_{G}(x), V(x)\right\rangle d x=0 .
\end{aligned}
$$

proving the lemma.

Proof of the Proposition 1. Let $X$ an eingenvector of div $\nabla$ on the space of the $G$-invariant vector fields associated to the eigenvalue $\lambda$. Then

$$
\int_{M}\langle-\operatorname{div} \nabla X, W\rangle d x=\lambda \int_{M}\langle X, W\rangle d x
$$

for all $G$-invariant vector field $W$. For proving that

$$
\int_{M}\langle-\operatorname{div} \nabla X, V\rangle d x=\lambda \int_{M}\langle X, V\rangle d x
$$

holds for any given vector field $V \in C^{\infty}(T M)$ we write $V=Z+V_{G}$ with $Z=V-V_{G}$ so that

$$
\int_{M}\langle-\operatorname{div} \nabla X, V\rangle d x=\int_{M}\langle-\operatorname{div} \nabla X, Z\rangle d x+\int_{M}\left\langle-\operatorname{div} \nabla X, V_{G}\right\rangle d x .
$$

Noting that $Z$ has zero $G$-mean and and that $\operatorname{div} \nabla X$ is a $G$-invariant because $X$ is we have, by Lemma 2 , that the first term of the hand side of the equality above is zero and then

$$
\begin{aligned}
\int_{M}\langle-\operatorname{div} \nabla X, V\rangle d x & =\int_{M}\left\langle-\operatorname{div} \nabla X, V_{G}\right\rangle d x=\lambda \int_{M}\left\langle X, V_{G}\right\rangle d x \\
& =\lambda \int_{M}\langle X, V-Z\rangle d x=\lambda \int_{M}\langle X, V\rangle d x
\end{aligned}
$$

proving the proposition. 
Remark 3 Considering a bi-invariant metric on the unit sphere $\mathbb{S}^{3}$ with the Lie group structure, $\mathbb{S}^{3}$ is a Lie subgroup of the isometry group $O(4)$ of $\mathbb{S}^{3}$. It follows from Proposition 1 that the $\mathbb{S}^{3}$-invariant vector fields are eigenvectors of the rough Laplacian of $\mathbb{S}^{3}$. Clearly the $\mathbb{S}^{3}$-invariant vector fields are the left (and right) invariant vector fields of $\mathbb{S}^{3}$. The orbits of a left invariant vector field constitute a Hopf fibration of $\mathbb{S}^{3}$.

We have that the energy of a left invariant vector field is 2 . Indeed, the Bochner-Yano formula for a vector field $X \in C^{\infty}\left(T \mathbb{S}^{3}\right)$, namely,

$$
\begin{aligned}
\int_{\mathbb{S}^{3}}|\nabla X|^{2} d x & =\int_{\mathbb{S}^{3}}\left(\operatorname{Ric}(X, X)+2|\operatorname{Kill}(X)|^{2}-(\operatorname{div} X)^{2}\right) d x \\
& =\int_{\mathbb{S}^{3}}\left(2|X|^{2}+2|\operatorname{Kill}(X)|^{2}-(\operatorname{div} X)^{2}\right) d x,
\end{aligned}
$$

where $\operatorname{Kill}(X)$ is the $(0,2)$-tensor

$$
\operatorname{Kill}(X)(U, V)=\frac{\left\langle\nabla_{U} X, V\right\rangle+\left\langle\nabla_{V} X, U\right\rangle}{2}, U, V \in C^{\infty}\left(T \mathbb{S}^{3}\right)
$$

(see [12]), when $X$ is a Killing field, since $\operatorname{Kill}(X) \equiv 0$ and $\operatorname{div} X \equiv 0$, gives

$$
F(X)=\frac{\int_{\mathbb{S}^{3}}|\nabla X|^{2} d x}{\int_{\mathbb{S}^{3}}|X|^{2} d x}=2 .
$$

As we will see in the next section, the infimum of $F$ on $\mathbb{S}^{n}$ is 1 , for all $n$. Thus, the $\mathbb{S}^{3}$-invariant vector fields do not realize the infimum of energy on $\mathbb{S}^{3}$.

\section{The infimum of the energy on a sphere}

In this section we determine the infimum of $F$ in the case that $M$ is a sphere. Denoting by $\mathbb{S}^{n}(1 / k)$ the sphere of radius $1 / k$ in $\mathbb{R}^{n+1}$ we prove:

Theorem 4 The infimum of $F$ on $C^{\infty}\left(T \mathbb{S}^{n}(1 / k)\right), n \geq 2$, is $k^{2}$, and is assumed by the orthogonal projection on $T \mathbb{S}^{n}(1 / k)$ of a constant nonzero vector field of $\mathbb{R}^{n+1}$. Moreover, a Killing field of $\mathbb{S}^{n}(1 / k)$ realizes the infimum $k^{2}$ of energy if and only if $n=2$.

We need some preliminary lemmas.

Lemma 5 Let $X$ be a vector field of $\mathbb{S}^{n}(1 / k)$ with zero $G-$ mean $\left(X_{G} \equiv 0\right)$, where $G$ is the isotropy subgroup of $\operatorname{Iso}\left(\mathbb{S}^{n}(1 / k)\right)$ that leaves fixed a point 
$v \in \mathbb{S}^{n}(1 / k)$. Then the function $f(p):=\langle X(p), v\rangle, p \in \mathbb{S}^{n}(1 / k)$, has zero mean in $\mathbb{S}^{n}(1 / k)$ that is,

$$
\int_{\mathbb{S}^{n}(1 / k)} f(x) d x=0
$$

Proof. Using the formula of coarea to integrate $f$ on $\mathbb{S}^{n}(1 / k)$ along the level sets of $h: \mathbb{S}^{n}(1 / k) \rightarrow \mathbb{R}, h(p)=d(p, v)$, where $d$ is the distance in $\mathbb{S}^{n}(1 / k)$, since $\|\operatorname{grad} h\|=1$, we have

$$
\int_{\mathbb{S}^{n}(1 / k)} f(x)=\int_{0}^{\pi}\left(\int_{h^{-1}(t)} f(x)\right) d t .
$$

We note that $h^{-1}(t)$ is a geodesic sphere $\mathbb{S}_{t}^{n-1}$ of $\mathbb{S}^{n}(1 / k)$ centered at $v$ and with radius $t$ and that these spheres are the orbits of $G$. Given $t \in$ $(0, \pi / k)$, choose $p \in h^{-1}(t)$, and denote by $H$ the subgroup of isotropy of $G$ at $p$. Let

$$
\psi: \frac{G}{H} \rightarrow \mathbb{S}_{t}^{n-1}
$$

be given by $\psi(g H)=g(p)$. We note that, up to a factor multiplying the metric of $G, \psi$ is an isometry. Setting

$$
\widetilde{f}:=f \circ \psi: \frac{G}{H} \rightarrow \mathbb{R}
$$

we then obtain

$$
\int_{h^{-1}(t)} f(x) d x=\int_{\frac{G}{H}} \widetilde{f}(g H) d(g H) .
$$

Let

$$
\phi: G \rightarrow \frac{G}{H}
$$

be the projection of $G$ over $G / H$, and set $\bar{f}:=\widetilde{f} \circ \phi$. Using the coarea formula to integrate $\bar{f}$ on $G$ along the fibers of $\phi$, we obtain

$$
\begin{aligned}
\int_{g \in G} \bar{f}(g) d g & =\int_{\frac{G}{H}}\left(\int_{g H} \frac{1}{\|\operatorname{Jac} \phi\|} \bar{f}(g h) d(g h)\right) d(g H) \\
& =\int_{\frac{G}{H}}\left(\int_{g H} \bar{f}(g h) d(g h)\right) d(g H) .
\end{aligned}
$$

Since $\bar{f}$ is constant on $g H, g \in G$, we get

$$
\int_{G} \bar{f}(g) d g=\operatorname{Vol}(H) \int_{\frac{G}{H}} \tilde{f}(g H) d(g H)
$$


or by (2)

$$
\int_{h^{-1}(t)} f(x) d x=\frac{1}{\operatorname{Vol}(H)} \int_{G} \bar{f}(g) d g
$$

But

$$
\begin{aligned}
\int_{G} \bar{f}(g) d g & =\int_{G} \tilde{f}(g H) d g \\
& =\int_{G}(f \circ \psi)(g H) d g=\int_{G} f(g(p)) d g \\
& =\int_{G}\langle X(g(p)), v\rangle d g=\int_{G}\left\langle d g_{p}^{-1} X(g(p)), d g_{p}^{-1} v\right\rangle d g \\
& =\int_{G}\left\langle d g_{p}^{-1} X(g(p)), v\right\rangle d g=\left\langle X_{G}(p), v\right\rangle=0 .
\end{aligned}
$$

Therefore from (3)

$$
\int_{h^{-1}(t)} f=0
$$

for any $t \in(0, \pi / k)$, and this, with (10), proves the lemma.

Lemma 6 Let $V \in C^{\infty}\left(T \mathbb{S}^{n}(1 / k)\right), n \geq 2$, with

$$
\int_{\mathbb{S}^{n}(1 / k)}|V|^{2}=1
$$

be given. Let $G$ be the isotropy subgroup of Iso $\left(\mathbb{S}^{n}(1 / k)\right)$ at some point of $\mathbb{S}^{n}(1 / k)$. If $V$ is nonzero and has zero $G-$ mean, then $F(V) \geq(n-1) k^{2}$.

Proof. Denote by $\nabla$ the Riemannian connection of $\mathbb{S}^{n}(1 / k)$. Write

$$
V=\sum_{l=1}^{n+1} a_{l} e_{l}
$$

where $\left\{e_{l}\right\}$ is an orthonormal basis of the $\mathbb{R}^{n+1}$. Fix $p \in \mathbb{S}^{n}(1 / k)$ and let $\left\{E_{j}\right\}$ be an orthonormal frame of $\mathbb{S}^{n}(1 / k)$ on a neighborhood of $p$. Then, for each $i$ we have

$$
\nabla_{E_{i}} V=\sum_{l=1}^{n+1} E_{i}\left(a_{l}\right) e_{l}-\left\langle V, E_{i}\right\rangle k p
$$


and thus

$$
\begin{aligned}
\langle\nabla V, \nabla V\rangle & =\sum_{i=1}^{n}\left\langle\nabla_{E_{i}} V, \nabla_{E_{i}} V\right\rangle \\
& =\sum_{i=1}^{n}\left(-\left\langle V, E_{i}\right\rangle^{2} k^{2}+\sum_{l=1}^{n+1}\left\langle E_{i}\left(a_{l}\right) e_{l}, E_{i}\left(a_{l}\right) e_{l}\right\rangle\right) \\
& =-k^{2}|V|^{2}+\sum_{l=1}^{n+1}\left|\operatorname{grad}\left(a_{l}\right)\right|^{2}
\end{aligned}
$$

We then have

$$
F(V)=-k^{2}+\sum_{l=1}^{n+1} \int_{\mathbb{S}^{n}(1 / k)}\left|\operatorname{grad}\left(a_{l}\right)\right|^{2} .
$$

By Lemma 5 the functions $a_{l}$ have zero mean on $\mathbb{S}^{n}(1 / k)$. Since the first positive eigenvalue of $\mathbb{S}^{n}(1 / k)$ is equal to $n k^{2}$, from Poincaré inequality we obtain

$$
\begin{aligned}
F(V) & \geq-k^{2}+n k^{2} \sum_{l=1}^{n+1} \int_{\mathbb{S}^{n}(1 / k)}\left|a_{l}\right|^{2} \\
& =-k^{2}+n k^{2} \int_{\mathbb{S}^{n}(1 / k)}|V|^{2}=(n-1) k^{2} .
\end{aligned}
$$

Lemma 7 Assume that a Lie subgroup $H$ of $O(n)=$ Iso $\left(\mathbb{S}^{n}(1 / k)\right)$ acts transitively on $\mathbb{S}^{n}(1 / k)$. Then

$$
\int_{H}\langle h u, v\rangle d h=0
$$

for any fixed vectors $u, v \in \mathbb{S}^{n}(1 / k)$.

Proof. Let $u, v \in \mathbb{S}^{n}(1 / k)$ be given. There is $g \in H$ such that $g(v)=-v$. Since the left translation $L_{g^{-1}}: H \rightarrow H, L_{g^{-1}}(h)=g^{-1} h$ is an orientation preserving isometry of $H$ we have

$$
\int_{H}\langle h u, v\rangle d h=\int_{H}\left\langle g^{-1} h u, v\right\rangle\left(L_{g^{-1}}\right)^{*} d h=\int_{H}\langle h u, g v\rangle d h=-\int_{H}\langle h u, v\rangle d h
$$

which proves (4). 
The next lemma will also be used to prove Theorem 12, We make use of the following terminology: An orbit of highest dimension of a compact Lie group $G$ acting on a compact manifold $M$ is called a principal orbit (except to the exceptional orbits, see [5]). We say that $G$ acts with cohomogeneity one if the principal orbits of $G$ have codimension 1. The principal orbits of $G$ foliates a open dense subset of $M$ whose complementary has zero $\operatorname{dim} M$-dimensional measure ([5]).

Lemma 8 Let $M^{n}$ be a compact, orientable Riemannian manifold. Let $G$ be a compact Lie subgroup of $\operatorname{Iso}(M)$ acting with cohomogeneity one on $M$. Assume moreover that the subgroup of isotropy of $G$ at any point of a principal orbit of $G$ acts transitively (by the derivative) on the spheres centered at origin of the tangent space of the orbit at the point. Then any $G$-invariant vector field is orthogonal to the principal orbits of $G$.

Proof. Let $p \in M$ be such that $G(p)$ is a principal orbit of $G$ and let $v \in T_{p} G(p)$ be any fixed vector. Since $X$ is $G$-invariant we have

$$
\langle X(p), v\rangle=\left\langle d g_{p}^{-1}(X(g(p))), v\right\rangle=\left\langle X(g(p)), d g_{p} v\right\rangle
$$

for all $g \in G$, so that

$$
\langle X(p), v\rangle=\frac{1}{\operatorname{Vol}(G)} \int_{G}\left\langle X(g(p)), d g_{p} v\right\rangle d g .
$$

Denoting by $H$ be the isotropy subgroup of $G$ at $p$ we have by coarea formula

$$
\int_{G}\left\langle X(g(p)), d g_{p} v\right\rangle d g=\int_{G / H}\left(\int_{g H}\left\langle X((g h)(p)), d(g h)_{p} v\right\rangle d(g h)\right) d(g H) .
$$

Moreover

$$
\begin{aligned}
\int_{g H}\left\langle X((g h)(p)), d(g h)_{p} v\right\rangle d(g h) & =\int_{H}\left\langle X(g(p)), d(g h)_{p} v\right\rangle d h \\
& =\int_{H}\left\langle d g_{p}^{-1} X(g(p)), d h_{p} v\right\rangle d h=0
\end{aligned}
$$

by the previous lemma, since the action $h \mapsto d h_{p}$ of $H$ on $T_{p} G(p)$ is transitive on the spheres of $T_{p} G(p)$. Then

$$
\langle X(p), v\rangle=\frac{1}{\operatorname{Vol}(G)} \int_{G}\left\langle X(g(p)), d g_{p} v\right\rangle d g=0 .
$$


This implies that $X(p) \in\left(T_{p} G(p)\right)^{\perp}$ since $v$ is arbitrary, concluding with the proof of the lemma.

Proof of Theorem 4. A calculation shows that if $X \in C^{\infty}\left(T \mathbb{S}^{n}(1 / k)\right)$ is the orthogonal projection on $T \mathbb{S}^{n}(1 / k)$ of a vector $v \in \mathbb{R}^{n+1}$ then $-\operatorname{div} \nabla X=$ $k^{2} X$. We will prove that $k^{2}$ is the infimum of $F$ and hence proving the theorem. We may assume that $v \in \mathbb{S}^{n}(1 / k)$.

Let $G$ be the isotropy subgroup of $\operatorname{Iso}\left(\mathbb{S}^{n}(1 / k)\right)$ at $v$ and $W \in C^{\infty}\left(T \mathbb{S}^{n}(1 / k)\right)$ assuming the infimum of $F$. It follows from Lemma 6 that if $W$ has zero $G$-mean, then $F(W) \geq(n-1) k^{2}$ and the theorem is proved in this case. We may then assume that $W_{G}$ is non zero. Since $\operatorname{div} W_{G}=(\operatorname{div} W)_{G}, F$ assumes its infimum at $W_{G}$ too.

By the Lemma 8, $W_{G}$ is orthogonal to the orbits of $G$, which are geodesic spheres centered at $v$. We then have $W_{G}=\left\langle W_{G}, \operatorname{grad} s\right\rangle \operatorname{grad} s$, where $s$ is the distance in $\mathbb{S}^{n}(1 / k)$ to $v$. Define $h \in C^{2}([0, \pi / k])$ by $h(t)=\left\langle W_{G}\right.$, grad $\left.s\right\rangle(x)$, where $x \in \mathbb{S}^{n}(1 / k)$ is such that $t=s(x)$. Since $W_{G}$ is $G$-invariant $h$ is well defined and we have $W_{G}=h(s) \operatorname{grad} s$.

If $\phi \in C^{2}([0, \pi / k])$ is a primitive of $h$ and $f: \mathbb{S}^{n}(1 / k) \rightarrow \mathbb{R}$ is defined by $f(x)=\phi(s(x))$ then we have

$$
\operatorname{grad} f=\phi^{\prime} \operatorname{grad} s=h \operatorname{grad} s=W_{G} .
$$

Applying Reilly's formula to $f$ (see [9]) we obtain

$$
\begin{aligned}
\int_{\mathbb{S}^{n}(1 / k)}(\Delta f)^{2} d x & =\int_{\mathbb{S}^{n}(1 / k)} \operatorname{Ric}_{\mathbb{S}^{n}(1 / k)}(\operatorname{grad} f, \operatorname{grad} f) d x \\
& +\int_{\mathbb{S}^{n}(1 / k)}|\operatorname{Hess}(f)|^{2} d x \\
& =\int_{\mathbb{S}^{n}(1 / k)} \operatorname{Ric}_{\mathbb{S}^{n}(1 / k)}\left(W_{G}, W_{G}\right) d x+\int_{\mathbb{S}^{n}(1 / k)}|\operatorname{Hess}(f)|^{2} d x
\end{aligned}
$$

where $\Delta f$ and $\mid$ Hess $(f) \mid$ are the Laplacian and the norm of the Hessian of $f$. Note that $|\operatorname{Hess}(f)|=\left|\nabla W_{G}\right|$ and then, since $(\Delta f)^{2} \leq n|\operatorname{Hess}(f)|^{2}$, assuming that $\left|W_{G}\right|_{L^{2}}=1$, we obtain

$$
\begin{aligned}
& (n-1) \int_{\mathbb{S}^{n}(1 / k)}\left|\nabla W_{G}\right|^{2} d x \\
& \geq \int_{\mathbb{S}^{n}(1 / k)}\left|W_{G}\right|^{2} \operatorname{Ric}_{\mathbb{S}^{n}(1 / k)}\left(\frac{W_{G}}{\left|W_{G}\right|}, \frac{W_{G}}{\left|W_{G}\right|}\right) d x \geq(n-1) k^{2} .
\end{aligned}
$$

It follows that $F\left(W_{G}\right) \geq k^{2}$, proving the first part of the theorem. For the last part, it follows from Bochner-Yano formula of Remark 3 that if $X$ is a 
Killing field in $\mathbb{S}^{n}(1 / k)$ then $F(X)=(n-1) k^{2}$ so that $F(X)=k^{2}$ if and only if $n=1$.

\section{The critical points of the energy on a rank 1 compact symmetric space}

In this section we prove:

Theorem 9 Let $M$ be a rank 1 compact symmetric space. Let $G$ be a compact Lie subgroup of $\operatorname{Iso}(M)$ that leaves pointwise fixed a totally geodesic submanifold of $M$ with dimension bigger than or equal to 1 . Then the all the critical points of the energy in $M$ are assumed by a $G$-invariant vector field.

If $V$ is a vector field on $M$ and if $h: M \rightarrow M$ is a diffeomorphism, we denote by $V^{h}$ the $h$-related vector field to $V$, that is, $V^{h}(p)=\left(d h_{p}\right)^{-1}(V(h(p))$, $p \in M$. We use the following lemma.

Lemma 10 Let $p \in M, v \in T_{p} M$ and $V \in C^{\infty}(T M)$ be given. Assume that $g(p)=p$ and $d g_{p}(v)=v$ for all $g \in G$. Given $q \in M$ assume that $h \in \operatorname{Iso}(M)$ is such that $h(p)=q$ and $d h_{q}^{-1}(V(q))=v$. Let $x_{n} \in M$ be a sequence converging to $p$. Then

$$
V^{h}(p)=\lim _{n \rightarrow \infty} \frac{\int_{G} d g_{x_{n}}^{-1}\left(V^{h}\left(g\left(x_{n}\right)\right)\right)}{\operatorname{Vol}\left(G\left(x_{n}\right)\right)}
$$

where $\operatorname{Vol}\left(G\left(x_{n}\right)\right)$ is the $k$-dimensional Hausdorff measure of $G\left(x_{n}\right), k=$ $\operatorname{dim} G\left(x_{n}\right) \geq 0$.

Proof. We will prove that

$$
\left\langle V^{h}(p), Z(p)\right\rangle=\left\langle\lim _{n \rightarrow \infty} \frac{\int_{G} d g_{x_{n}}^{-1}\left(V^{h}\left(g\left(x_{n}\right)\right)\right)}{\operatorname{Vol}\left(G\left(x_{n}\right)\right)}, Z(p)\right\rangle, Z \in C^{\infty}(T M) .
$$

Given $Z \in C^{\infty}(T M)$ we have, for a given $n$,

$$
\left\langle\frac{\int_{G} d g_{x_{n}}^{-1}\left(V^{h}\left(g\left(x_{n}\right)\right)\right)}{\operatorname{Vol}\left(G\left(x_{n}\right)\right)}, Z\left(x_{n}\right)\right\rangle=\frac{\int_{G}\left\langle d g_{x_{n}}^{-1}\left(V^{h}\left(g\left(x_{n}\right)\right)\right), Z\left(x_{n}\right)\right\rangle}{\operatorname{Vol}\left(G\left(x_{n}\right)\right)}
$$


so that

$$
\begin{aligned}
\inf _{g \in G}\left\langle d g_{x_{n}}^{-1}\left(V^{h}\left(g\left(x_{n}\right)\right)\right), Z\left(x_{n}\right)\right\rangle & \leq \frac{\int_{G}\left\langle d g_{x_{n}}^{-1}\left(V^{h}\left(g\left(x_{n}\right)\right)\right), Z\left(x_{n}\right)\right\rangle \omega}{\operatorname{Vol}\left(G\left(x_{n}\right)\right)} \\
& \leq \sup _{g \in G}\left\langle d g_{x_{n}}^{-1}\left(V^{h}\left(g\left(x_{n}\right)\right)\right), Z\left(x_{n}\right)\right\rangle .
\end{aligned}
$$

Letting $n \rightarrow \infty$ we obtain

$$
\begin{aligned}
\inf _{g \in G}\left\langle d g_{p}^{-1}\left(V^{h}(g(p))\right), Z(p)\right\rangle & \leq\left\langle\lim _{n \rightarrow \infty} \frac{\int_{G} d g_{x_{n}}^{-1}\left(V^{h}\left(g\left(x_{n}\right)\right) d g\right.}{\operatorname{Vol}\left(G\left(x_{n}\right)\right)}, Z(p)\right\rangle \\
& \leq \sup _{g \in G}\left\langle d g_{p}^{-1}\left(V^{h}(g(p))\right), Z(p)\right\rangle,
\end{aligned}
$$

and, since $g(p)=p$,

$$
\begin{aligned}
\inf _{g \in G}\left\langle d g_{p}^{-1}\left(V^{h}(p)\right), Z(p)\right\rangle & \leq\left\langle\lim _{n \rightarrow \infty} \frac{\int_{G} d g_{x_{n}}^{-1}\left(V^{h}\left(g\left(x_{n}\right)\right)\right) d g}{\operatorname{Vol}\left(G\left(x_{n}\right)\right)}, Z(p)\right\rangle \\
& \leq \sup _{g \in G}\left\langle d g_{p}^{-1}\left(V^{h}(p)\right), Z(p)\right\rangle .
\end{aligned}
$$

Since

$$
d g_{p}^{-1}\left(V^{h}(p)\right)=d g_{p}^{-1}(v)=v=V^{h}(p)
$$

for all $g \in G$ it follows that

$$
\left\langle V^{h}(p), Z(p)\right\rangle \leq\left\langle\lim _{n \rightarrow \infty} \frac{\int_{G} d g_{x_{n}}^{-1}\left(V^{h}\left(g\left(x_{n}\right)\right)\right) d g}{\operatorname{Vol}\left(G\left(x_{n}\right)\right)}, Z(p)\right\rangle \leq\left\langle V^{h}(p), Z(p)\right\rangle
$$

proving the lemma.

Proof of Theorem 9. Let $V \in C^{\infty}(T M)$ be a critical point of $F$ with unit $L^{2}$-norm. Since $V$ is non zero, there is $q \in M$ such that $V(q) \neq 0$. Let $G$ be a Lie subgroup of $\operatorname{Iso}(M)$ that leaves pointwise fixed a totally geodesic submanifold $T$ of $M, \operatorname{dim} T \geq 1$. Choose a $p \in T$ and a nonzero vector $v \in T_{p} T$. We have $g(p)=p$ for all $g \in G$ and we claim that $d g_{p}(v)=v$ for all $g \in G$ too. Up to a multiplication of $v$ by a positive real number, we may assume that $v$ is contained on a normal geodesic ball of $T_{p} T$. Set $w=d g_{p}(v)$. Then, since $\exp _{p} v \in T$, where $\exp _{p}$ it the Riemannian exponential, we have

$$
\exp _{p} v=g\left(\exp _{p} v\right)=\exp _{g(p)} d g_{p}(v)=\exp _{p} w
$$


and then $v=w$.

Since $M$ is a compact rank 1 symmetric space, there is an isometry $h$ of the $M$ such that $h(p)=q$ and $d h_{p}^{-1}(V(q))=v$. Let $V^{h}$ be the vector field $h$-related to $V$. We claim that $V_{G}^{h}$ is not identically zero. Indeed, taking a sequence $x_{n} \in M$ converging to $p$, we have, by the Lemma 10

$$
V^{h}(p)=\lim _{n \rightarrow \infty} \frac{\int_{G} d g_{x_{n}}^{-1}\left(V^{h}\left(g\left(x_{n}\right)\right)\right)}{\operatorname{Vol}\left(G\left(x_{n}\right)\right)}=\lim _{n \rightarrow \infty} \frac{V_{G}^{h}\left(x_{n}\right)}{\operatorname{Vol}\left(G\left(x_{n}\right)\right)} .
$$

Hence, if $V_{G}^{h} \equiv 0$ then $V^{h}(p)=0$, a contradiction! This proves the theorem.

\section{A characterization of spheres}

Rotationally symmetric manifolds are well known and much used as models on comparison theorems on Geometric Analysis. We consider here a generalization of such manifolds which we call two point symmetric manifolds:

Definition 11 We say that a Riemannian $n$-dimensional manifold $M$, $n \geq 2$, is two point symmetric with center $p \in M$ if the isotropy subgroup $G:=\operatorname{Iso}_{p}(M)$ of the isometry group of $M$ at $p$ is isomorphic to the isotropy subgroup of the isometry group of a two point homogeneous space $S$ at any point in $S$.

We note that the above definition is a natural generalization of the way used in [3] to define rotationally symmetric spaces. We also observe that this definition is equivalent to the requirement that given points $p_{1}, p_{2}, p_{3}, p_{4} \in M$ that belong to a common geodesic sphere of $M$ centered at $p$, if $d\left(p_{1}, p_{2}\right)=$ $d\left(p_{3}, p_{4}\right)$, where $d$ is the Riemannian distance in $M$, then there is an isometry $i \in G$ such that $i\left(p_{1}\right)=p_{3}$ and $i\left(p_{2}\right)=p_{4}$ (see [1, [4]). It is also known that this two point homogenous characterization of $M$ around $p$ is equivalent to the isotropy of $G$ at any point $x \in M$ of a principal orbit $G(x)$ of $G$ acting transitively on the Euclidean spheres centered at the origin of $T_{x} G(x)$ ([4]).

We also recall that a two point homogeneous space is isometric to a rank 1 symmetric space. Accordingly to the symmetric space classification, it follows that a Riemannian manifold $M$ is two point homogenous with center $p \in M$ if $\operatorname{Iso}_{p}(M)$ is isomorphic to one of the following Lie groups: $O(n), U(1) \times U(n), S p(1) \times S p(n)$ or $\operatorname{Spin}(9)$ (see [4]). When $\operatorname{Iso}_{p}(M)=O(n)$ the space $M$ is rotationally symmetric. 
Theorem 12 Let $M^{n}$ be a compact two point symmetric space with center $p, n \geq 2$, with positive Ricci curvature $\operatorname{Ric}_{M}$ and assume that $\operatorname{Ric}_{M} \geq(n-$ $1) k^{2}, k>0$. Set $G=\operatorname{Iso}_{p}(M)$. Then the infimum of $F$ on the $G$-invariant vector fields is bigger than or equal to $k^{2}$ and the equality holds if and only if $M$ is a sphere of radius $1 / k$.

Proof. Denote by $s: M \rightarrow \mathbb{R}$ the distance in $M$ to $p$. Note that the level sets of $s$ are geodesic spheres centered at $p$ and that the mean curvature and the norm of the second fundamental form of these geodesic spheres depend only on $s$. Set $l=\max s$.

Let $V \in C^{\infty}(T M)$ a $G$-invariant vector field such that $F(V)$ is the positive infimum of $F$ on the space of $G$-invariant vector fields. Since the subgroup of isotropy of $G$ at a point $p$ of a principal orbit of $G$ acts transitively (by the derivative) on the spheres centered at origin of $T_{p} G(p)$ it follows from Lemma 8 that $V$ may be written on the form $V=\langle V, \operatorname{grad} s\rangle \operatorname{grad}$. Define $h, f$ and $\phi$ as in Theorem 4 . The same reasoning used in this theorem allows us to conclude that $F(V) \geq k^{2}$, proving the first part of the theorem. By Theorem 4 we know that if $M$ is a sphere of radius $1 / k$ then $F(V)=k^{2}$. We now prove the converse. Thus, assume that $F(V)=k^{2}$. From the inequality obtained from Reilly's formula in Theorem 4 we obtain

$$
(\Delta f)^{2}=n|\operatorname{Hess}(f)|^{2} .
$$

Given $s \in(0, l)$ denote by $|B|(s)$ and $H(s)$ the norm of the second fundamental form and the mean curvature of the geodesic sphere at a distance $s$ of $p$, with respect to the unit normal vector pointing to the center $p$. Since $f=\phi \circ s$, straightforward calculations give

$$
(\Delta f)^{2}=\left(\phi^{\prime \prime}\right)^{2}-2(n-1) \phi^{\prime \prime} \phi^{\prime} H+(n-1)^{2}\left(\phi^{\prime}\right)^{2} H^{2}
$$

and

$$
|\operatorname{Hess}(f)|^{2}=\left(\phi^{\prime \prime}\right)^{2}+\left(\phi^{\prime}\right)^{2}|B|^{2} .
$$

Then (5) is equivalent to

$$
\left(\phi^{\prime \prime}+H \phi^{\prime}\right)^{2}=\left(\phi^{\prime}\right)^{2}\left[-\frac{n|B|^{2}}{n-1}+n H^{2}\right]
$$

which implies that

$$
-\frac{|B|^{2}}{n-1}+H^{2} \geq 0
$$


since $\phi^{\prime}$ cannot be identically zero (otherwise $V$ would be, which is not the case). But, denoting by $\lambda_{i}$ the principal curvatures of the geodesic spheres, we have

$$
\begin{aligned}
-\frac{|B|^{2}}{n-1}+H^{2} & =\frac{-\left(\sum_{i=1}^{n-1} \lambda_{i}^{2}\right)}{n-1}+\frac{\left(\sum_{j=1}^{n-1} \lambda_{j}\right)^{2}}{(n-1)^{2}} \\
& =-\sum_{i, j=1, i<j}^{n}\left(\lambda_{i}-\lambda_{j}\right)^{2} \leq 0
\end{aligned}
$$

so that

$$
-\frac{|B|^{2}}{n-1}+H^{2}=0
$$

and, from (6),

$$
\phi^{\prime \prime}+H \phi^{\prime}=0
$$

Since

$$
V=\operatorname{grad} f=(h \circ s) \operatorname{grad} s
$$

and $V$ is an infimum of $F$ we have

$$
-\operatorname{div} \nabla(h(s) \operatorname{grad} s)=k^{2} h(s) \operatorname{grad} s .
$$

A calculation gives

$$
\operatorname{div} \nabla(h \operatorname{grad} s)=\left(h^{\prime \prime}(s)-(n-1) H(s) h^{\prime}(s)-|B|^{2}(s) h(s)\right) \operatorname{grad} s
$$

so that

$$
h^{\prime \prime}-(n-1) H h^{\prime}-|B|^{2} h=-k^{2} h .
$$

But from (8) we have

$$
h^{\prime \prime}-(n-1) H h^{\prime}-|B|^{2} h=h^{\prime \prime}+(n-1)\left[H^{2}-|B|^{2} /(n-1)\right] h=h^{\prime \prime}
$$

and hence

$$
h^{\prime \prime}(s)=-k^{2} h(s) .
$$

It follows that $h$ is of the form

$$
h(s)=A \cos (s k)+B \sin (k s)
$$


and, since $\phi^{\prime}=h$,

$$
\phi(s)=\frac{A}{k} \cos (s k)+\frac{B}{k} \sin (k s) .
$$

From $f(x)=\phi(s(x))$ we then obtain using (8)

$$
\Delta f=\phi^{\prime \prime}+\phi^{\prime} \Delta s=\phi^{\prime \prime}-(n-1) H \phi^{\prime}=\phi^{\prime \prime}+(n-1) \phi^{\prime \prime}=n \phi^{\prime \prime}=-n k^{2} \phi=-n k^{2} f \text {. }
$$

Hence $n k^{2}$ is a eigenvalue of the usual Laplacian. Under the hypothesis $\operatorname{Ric}_{M} \geq(n-1) k^{2}$ this implies that $M$ is a sphere of radius $1 / k([])$, finishing the proof of the theorem.

\section{References}

[1] I. Chavel: "On Riemannian symmetric spaces of rank one", Advances in Mathematics, V. 4, N. 3, 1970, 236-263

[2] L. Bonorino, J. Ripoll: "The spectrum of the rough Laplacian on rotationally symmetric surfaces", preprint

[3] H. I. Choi: "Asymptotic Dirichlet problems for harmonic functions on Riemannian manifolds". Trans. Am. Math. Soc., 281 (2): 691-716, 1984

[4] S. Helgason: "Differential Geometry, Lie Groups, and Symmetric Spaces"', Graduate Texts in Mathematics, Vol 34, American Mathematical Society

[5] W. Y. Hsiang, B. Lawson: "Minimal submanifolds of low cohomogeneity", Journal of Differential Geometry, Vol 5, N. 1-2 , 1971, 1-38.

[6] J. Jost: "Postmodern Analysis", Universitext, Second Edition, Springer-Verlag, 2003

[7] J. Milnor: "Curvatures of left invariant metrics on lie groups", Advances in Mathematics, Vol. 21, N. 3, 1976, 293-329

[8] M. Obata: "Certain Condition for a Riemannian manifold to be isometric with a sphere", Math. Soc. Japan 14 (1962), 333-340.

[9] R. C. Reilly: "Applications of the Hessian operator in a Riemannian manifold", Indiana Univ. Math. J., 26, 1977, 459-472 
[10] S. Rosenberg, "The Laplacian on a Riemannian Manifold", Vol. 31, London Mathematical Society Student Texts, Cambridge Univ. Press, 1997.

[11] H. Urakawa: "Calculus of variations and harmonic maps", Translations of Mathematical Monographs, Vol 132, Providence, Rhode Island, AMS, 1993

[12] K. Yano: "Integral formulas in Riemannian geometry", New York: Marcel Dekker, 1970. 